\title{
CCDs IN ACTIVE ACQUISITION SYSTEMS
}

\author{
A. Blecha \\ Observatoire de Genève
}

\begin{abstract}
The use of CCD detectors as elements of an active acquisition system is reviewed. In such systems, the CCD image acquisition, data-analysis and the instrument and telescope controls are no longer separated elements whose actions are coordinated by an astronomer and/or operator, but are parts of a global system. The interaction between incoming data (nature of the object, registered flux, current PSF and atmospheric transmission), observer's requirement $(\mathrm{S} / \mathrm{N}$, spatial and temporal resolution) and forthcoming $\mathrm{CCD}$ exposure characteristics (CCD preparation, exposure time, read-out parameters) is examined. The requirements for the $\mathrm{CCD}$ electronics, data acquisition system are evaluated and examples of recent application in imaging, spectroscopy, photometry and auxiliary equipment are given. An attempt is made to analyze future technological trends and possible bottlenecks in such systems and to propose simple rules to adopt when designing CCD hardware and software.
\end{abstract}

\section{INTRODUCTION}

The CCDs, and electronic imaging devices in general, represent the modern version of photography and as such inherited the overwhelming majority of roles previously assumed by it. The need for a linear and efficient solid-state integrating detector was so obvious, that since the very first CCD system, and despite some drawbacks compared to photography, the success was rapid and irreversible. While the immediate availability of data was soon used for rapid visual inspection (and this despite the necessity to resort to very expensive special hardware equipment) it took some time before a more elaborate interaction between recorded data and instrumentation itself was introduced. Several technical, scientific and even psychological factors contributed to slow down the evolution towards an acquisition system with a direct feed-back in the acquisition loop. The principal sources of limitations were the hardwired unprogrammable electronics, the insufficient and expensive computing resources, the lack of standard basic data-acquisition SW layer and immature data-processing SW.

Each of the limiting factors is subject to evolution, but not all of them are evolving on the same time-scale. While computing resources are growing by a factor of approximately two per year, the requirements driven by the size of CCD chips follow a much slower trend. It took approximately 20 years to go from $400^{2}$ chips to $2048^{2}$ commercially available chips giving a yearly growth of 1.17 and even if we consider a size as large as $4000^{2}$, as available today (mosaic of four two-edge buttable $2000^{2}$ chips), the growth factor would be only 1.25 . The actual trend in electronics is towards fully programmable devices, removing another limitation from the above list. As a consequence of this evolution, the technical limitations hindering the use of the $\mathrm{CCD}$ as an active element of an acquisition system tend to disappear. The purpose of my paper is to review some existing active applications and evaluate how far we have gone in this 
direction and list requirements for the design of the control system (CCD controllers and software).

\section{ACTIVE ACQUISITION SYSTEMS}

\subsection{What is an Active Acquisition System?}

From the viewpoint of the interaction between data and the acquisition system we could distinguish two types of acquisition:

- The passive system merely records the data. No feedback mechanism between the recorded data and observing parameters is built into such a system. The acquisition of a CCD image and data-storing, even with sophisticated pipe-line processing, remains passive as long as there is no automatic interaction between what the pipe-line may see and the observing process. Such a system may extract automatically something which is useful for observation, but does not interact directly with the observing process.

- As soon as the result of data-processing directly influences (with or without observers acknowledgment) the observing process (what is done next), the system is close-looped or active.

A very simple example is the control of CCD saturation. In a good passive system, some automatic warning is generated if saturation is reached. Such warning may be provided through image display (saturated pixels displayed in a special color), by a numerical qualifier, special sound or any mixture of these. The warning is the outcome of the operation done on already acquired data and if these are unacceptable, the observation must be repeated. No corrective action is offered. In an active system, if saturation control is turned on, the system will take an extra preliminary snapshot of the field, and check for the saturation conditions of the exposure to be made. If saturation is predicted, the system proposes a corrected set of exposure parameters (shorter exposure time, lower gain, ...). The specifications of saturation conditions may be very general (\% of the saturated area, with or without removal of cosmic-rays, only central area scanned, ....).

\subsection{Existing Realizations}

The majority of existing active systems are related to robotic telescopes. Automatic Photometric Telescopes (APTs) have been in routine operation for several years (Genet et al. 1987, Bruton et al. 1989, Nelson and Zeilik 1990, Rodono and Cutispoto 1992) but have been built and operated with photoelectric photometers and not with CCDs.

The CCD based APTs applications are still rare. Papers presented during the meeting of the A.S.P. (Filippenko 1991) in a chapter devoted to CCD imaging, describe several interesting applications of active systems. Some systems are devoted to a specific scientific program. The Berkeley Automated SN Search (Perlmutter et al. 1992) uses the result of image processing of the current exposure to decide whether the follow up exposure has to be taken. The Explosive Transient Camera (Vanderspek et al. 1991) detects the new object in the monitored portion of the sky within a time-scale of a few seconds and sends the information to a fast moving 
photometric telescope to proceed to the measurement of the event. Other systems such as the Australian Automated Patrol Telescope described by Brooks (Brooks 1990) or The Berkeley Automatic Imaging Telescope (BAIT) presented by Treffers (Richmond et al. 1993) are more versatile systems designed more or less to be general-purpose imaging instruments.

Though first to use the active approach, the robotic telescopes are not, paradoxically, best examples of active systems. Constraints of unattended operation makes them suitable for routine work, but the requirement that all decisions should be taken automatically (absence of the observer) leads to a very limited number of rather static situations where the active system is used. The best laboratory for the active system is a normally operated telescope, which, under some conditions, could be run as a robotic telescope but remains a general purpose system with active system features. The experience from the operation of the CCD photometric Swiss facility at La Silla is an example of such an approach.

\section{SWISS PHOTOMETRIC TELESCOPE AT LA SILLA}

\subsection{Description of the system}

For the last 16 years the Geneva Observatory has been operating at La Silla Observatory a 70-cm Cassegrain telescope equipped with a photoelectric photometer (Burnet 1976) fitted with seven-color Geneva filters (Rufener and Nicolet 1988). In 1991 a CCD photometric camera was permanently attached to the Nasmyth focus to extend the Geneva system to a CCD detector and to serve as the test bench for the project of a new active-system 1.2-m telescope. The CCD camera (Blecha et al. 1991), built around the Astromed 2200 detector fitted with an EEV-GEC p8603 chip is equipped with filters matched to Geneva photometric passbands augmented with $\mathrm{R}$ and I filters (Blecha et al. 1994). The CCD camera has been integrated in the general acquisition environment which controls the telescope and instrument functions. Furthermore, the acquisition system has direct access to image and data-processing and display, Guide Star Catalogue (GSC) database (Lasker et al. 1990) and display, the commercial database $(/ \mathrm{rdb})$ and the Unix shell.

The acquisition system has been designed to enable easy integration of heterogeneous elements. Existing facilities (telescope) and new commercial hardware (CCD detector) have to be interfaced with a general computer environment and various software services in a way to make the developed system largely independent of the hardware and third party software. The central piece of the control system is the shell-like interpreter/pseudo-compiler (Weber 1992, 1993, Blecha and Weber 1992) that ensures coordination between all elements involved. All elements are controlled through server-client architecture and the programming is done through several layers of interpreted language. The user is interacting with the upper layer either via permissive (shortcuts, line completion, parameter defaults ...) line commands or through menus. Such a system allows almost unlimited active functions with an extremely efficient debugging and testing cycle. All active-system features have been implemented during normal observing runs. Since all logic of the system is coded in high-level language (plain ASCII files), it is possible to switch instantly from the development environment to the operational one. The software design is based on the following principles:

- All services are implemented as genuine servers. Each server understands and executes a set of commands and returns results to the client but it is a completely 
independent process(es).

- All hardware and software functions are (also) directly callable from the high-level interpreted language.

- The execution of the shell scripts is preceded by a pseudo compilation during which formal errors are detected. This increases the speed of development and implementation of new features.

- The control of errors and interruptions is supported and handled by the shell script.

The same software system is used for the full on-line reduction of the data from ELODIE, the new compact fiber-fed echelle spectrograph at St. Michel Observatory (Queloz 1995). below.

At La Silla, several active-system functions are implemented. Some examples are given

\subsection{Flatfield Acquisition}

This is the simplest (though not the less valuable) implementation of active use of the $\mathrm{CCD}$. The everyday regular flatfielding on the sky in several filters is something that requires rather expert manipulation (see for example Tyson and Gal 1993) and in our case (nine filters) it is almost impossible to carry out this task properly without some automatic procedure.

To obtain high quality flatfields the exposure time should be at least ten sec (to avoid shutter modulation), the exposure should be made close to a given position in the local sky (elevation and azimuth) and in a selected area (no stars brighter that $\mathrm{m}=15$ ) and the flux per pixel in all filters should be as close as possible to a specified value. To satisfy the above requirements, the order of filters, the exposure scheduling and duration may be adjusted.

Fig. 1 shows the flow chart of the acquisition. After the initial adjustments the system loops in the flux-meter mode taking short exposures through reference filter in the reference area $\left(50^{2}\right.$ pixel in central area of the $\left.\mathrm{CCD}\right)$ and estimating the required exposure time until the optimal conditions are reached. Then the FF exposures are made, each time with the net result being a very regular and unattended exposure in all filters. The level of exposure from night to night and filter to filter does not vary more reprogrammed many times, the only genuine dynamic parameter is the exposure time.

\subsection{Automatic Measurement of a Standard Star}

A good distribution of standard stars within the night and within the airmass range is crucial for the quality of the photometry. Usually only one photometric standard star is available within the field and it is neither efficient nor necessary to read the whole CCD. The observing sequence implements the search for the standard star in the database, the telescope pointing, the display of the GSC sky-map, the automatic centering (if required) and the programming of the CCD windowed read-out (window position, and size). The current PSF is used for the determination of the window size. The photometry is done for each filter and 


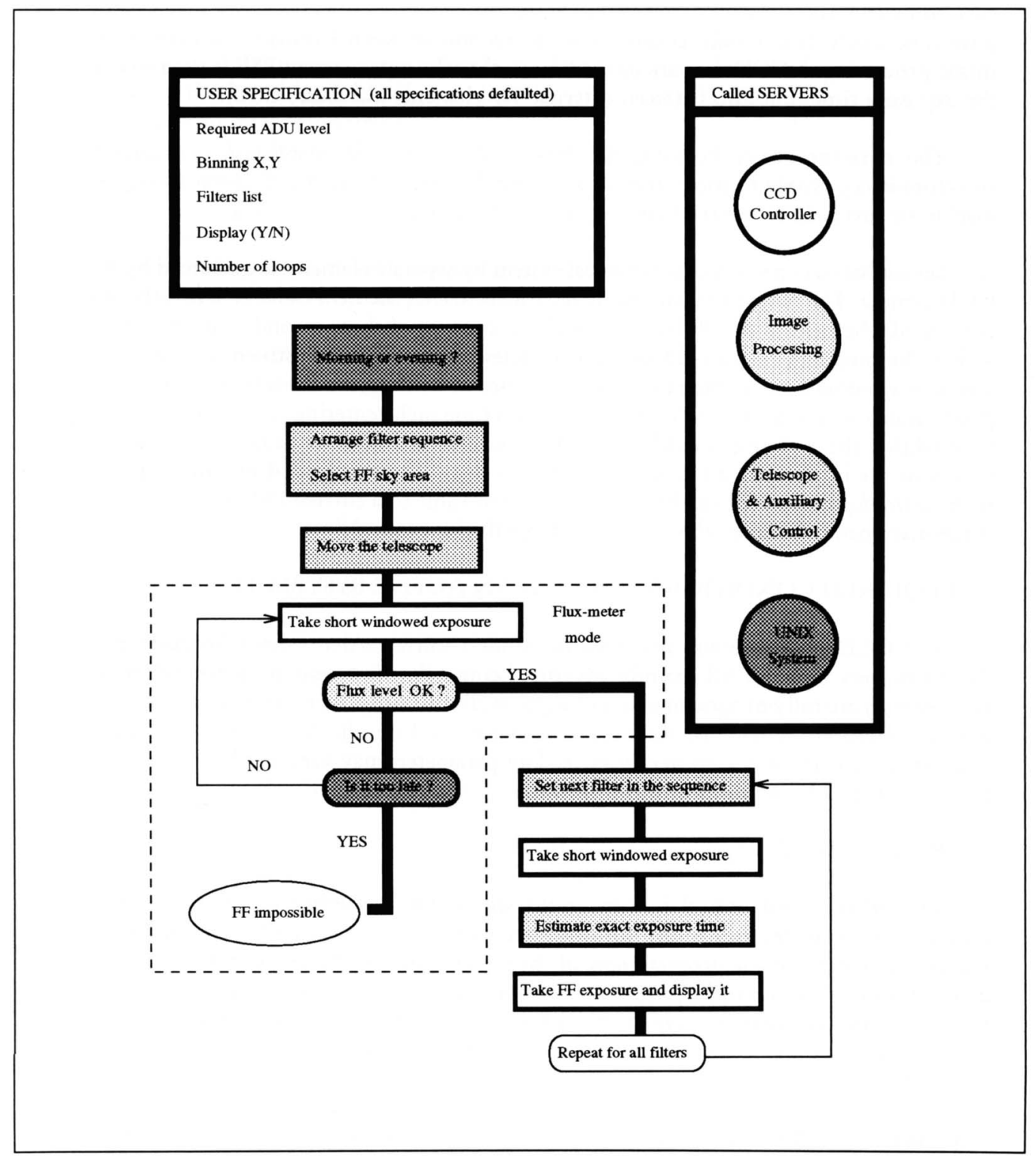

Fig. 1. Automatic flatfielding

repeated if more than one sample is required. Data are stored in a single image-like patched structure similar to that described by Wei (Wei et al. 1990) and during the current exposure, the previous exposure is analyzed. For each exposure the display is updated (patched with the 
current image), the airmass corrected flux is computed and several checks are made (saturation, position). There is not only considerable interaction between hardware elements (results of image processing of CCD data are used to move the telescope, current PSF is used to constrain the exposure time) but also between external SW elements (database, GSC).

The correspondence between the GSC and the sky is established permanently and therefore it is possible to move the telescope by designing the center of the field on the GSC display or inversely to see the GSC sky-map in the currently pointed area.

Several services provided in a classical system by separate elements are assumed by the same CCD camera. The most successful auxiliary role is that of the field camera. When the telescope is pointed, the field is usually shown rapidly (exposure of three seconds without filter, three $\mathrm{x}$ three binning and fastest readout) and the telescope position adjustment is made in a most simple way either by designing the object to center or by dragging the selected object to a given position in a way similar to CAD software. Each time such centering is done, the database used to establish the pointing model is updated. Several maintenance, auxiliary, and watchdog functions are implemented through the active system. The example of one interesting feature is the watchdog comparing the ESO seeing monitor value to a current PSF that issues a warning if the discrepancy between the two is too large (bad focussing).

\section{REQUIRED CONDITIONS TO BUILD AN ACTIVE SYSTEM THAT USES A CCD}

The CCD hardware and firmware (or system software that cannot be easily modified) should be very flexible. All recently advertised controllers (see session on controllers in these proceedings) are fully programmable and support all standard features that are required (flexible binning, multiples windowed readout, variable gain and speed). What makes the difference is how many readout parameters may vary readout parameters may vary and how easily they may be controlled and modified.

\subsection{Read-Out Speed}

One of the most critical features is the speed. The capability of the system to switch rapidly from one readout mode to another could be characterized by the minimum time required to carry out the programming of the $\mathrm{CCD}$ controller for a given mode including the down-loading of the controller program if necessary, the readout of the $\mathrm{CDD}$ and the transfer of the data into the memory where the control system may use them and finally the data conversion including the formatting and un-scrambling in multi-port or multi-window mode. The time to accomplish this cycle defines a shortest time-constant of any close-loop activity. Several such minimum turnaround times should be examined depending on the application. Typically, we will consider the fastest readout time of the full frame with degraded spatial (binning) and intensity (bits/sample) resolution, the readout time of a small window with the lowest readout noise (this time should be independent of the position of the window) and the time necessary to flush the CCD after the exposure of a bright object including the eventual setting time.

It is a widespread belief that the slow CCD read-out is mainly due to the requirement to keep the read-out noise very low. This is true only in a very limited number of applications working with a low $\mathrm{S} / \mathrm{N}$ image (high resolution spectroscopy of faint objects for example). As 
soon as the $\mathrm{S} / \mathrm{N}$ is more than ten, the read-out noise of a modern CCD system becomes negligible compared to other sources of noise (shot-noise, background, ...) and may be increased to some extent if it brings some advantage. And it does. The read-out noise obeys approximately the logarithmic law (for ex. Janesick et al. 1988) and the read-out noise (RMS) obtained at a given integration time $t_{r d}$ could be written as $R M S \approx 1 / t_{r d}{ }^{0.4}$. Increasing the speed by a factor of six only doubles the read-out noise while a factor of 15 in speed leads to a tripled read-out noise. The above relation is a good approximation in the interval $20-0.1 \mu \mathrm{sec}$.

It is possible to read some chips (Lincoln Lab.) in correlated-double-sampling mode with the speed of five Mega-samples/sec and RMS of read-out noise 10e. For more classical chips the read-out noise figure has to be multiplied by a factor of two to three.

None of the available controllers is fast enough to handle several-megapixel/sec at 16-bit resolution. The most critical element is the video chain, but probably the whole controller will have to be redesigned.

\subsection{Programming of the CCD}

Though all recent controllers are programmable, several levels should be distinguished:

-Level 1 - Laboratory programming.

In principle the controller is programmable but this is done almost by hand-coding. In such a system almost one-to-one correspondence between the controller state and the instruction exists. It does not make a difference whether the binary or symbolic coding is used.

- Level 2 - High performance parameterization.

The controller understands a high-level control command that specifies all readout parameters. All usual parameters such as binning factor, time-constants, gains or windowing layout are adjustable. Un-scrambling of the data-flow in windowed or multi-port readout is done by the controller and the high-level user has only to define parameters. This is the genuine server. The system may be fast and easy to use. Since only parameters are changed the system is relatively safe, but has some limits. Not all readout modes that the hardware could possibly implement may be supported.

\section{- Level 3 - Programming operational environment.}

The controller is designed as an dynamical instrument and efficient development tools are provided. A genuine high-level programming language is provided that enables us to describe waveforms, data flow control and electronics setup into a source file which is compiled into a program executable by a controller. During the execution the Level 2 control may be used. This approach is the most versatile and provides both the laboratory and operational level of programming.

For an active system level 1 programmability is insufficient. At least level 2 should be 
available and for more ambitious projects level 3 would be preferred.

Starting from 1990 an interesting development has been made by the CTIO CCD group (Smith 1994). A general Waveform Definition Language (WDL) has been adopted to program the CTIO Array CCD controller. The symbolic C-syntax language has been used to fully describe the 24-bit sequencer waveform. The source program(s) (ASCII-files) are processed using the standard cPp C-preprocessor then compiled. The result is still an ASCII file and is converted to the executable binary data only during the (down)load. Though the compiler produces the hardware specific output the WDL is powerful enough to generate the sequence for virtually any similar electronics. This system is indeed complemented by a hardware-dependent run-time system controlling the down-loading, chaining and execution of waveforms. The system does not implement genuine run-time parameters, but provides means to rapidly chain pre-compiled waveform segments.

\subsection{Shell Issue and High-Level Programming}

The discussion of the general software issue is beyond the scope of this contribution. Let us mention that almost all recent projects of large telescopes use or foresee to some extent an active control system with a sort of shell as a central piece of software. Several relevant papers were presented during the recent S.P.I.E. Symposium on Telescopes and Instrumentation for the $21^{\text {st }}$ Century, 13 - 18 March 1994, Kona, Hawaii and many documents have been produced by ESO in the framework of the VLT project.

The specific discussion below is relevant to the UNIX implementation.

\subsubsection{Shell Performance}

The speed of the CCD controller would be useless if the data are not available rapidly to the processing. In principle any data-processing environment able to supply basic data processing functions may be adopted as a shell. Both, the public domain (various UNIX shells, IRAF, MIDAS) or commercial packages (IDL, Matlab) are possible candidates. Once again, the critical aspect of the choice lies rather in the performance than in the ability to carry out a given task. The most critical aspect is the data input. The system with shared-memory buffers will be the more efficient since the incoming CCD data may be written directly to the working buffer. The performance may nevertheless be poor if the CCD readout and data transfer between the controller and the data analysis system are not done simultaneously. The system with internal working buffers is better than one which relies only on files since often in timecritical operations images are not stored permanently. The data format (encoding) supported by the data-processing system may be relevant. Since the native format of the CCD data is usually a 16-bit integer in many operations it is not useful to convert it in the floating format. The on-the-fly conversion may be the other alternative.

Nevertheless the time-critical issue is relevant to some limited and foreseeable tasks. The most interesting feature of the active system, the ability to combine and coordinate the high-level data reduction tasks with the control of data acquisition, may accommodate with some overhead incurred due to the less efficient, but more standard or easy-to-implement data communication (pipes, files, message systems). Other aspects may then be more important (available applications, command language, error handling, ...). Since the system should remain open, the 
two-way (back and forth) communication with the external world should be possible with no loss of information (conservation of keywords, descriptors, ...!).

The shell programs should be easy to program, debug and test. All categories of users (astronomer, maintenance staff, developers) should be able to write and modify such programs. Many interesting applications concern technical development and maintenance.

\section{CONCLUSIONS}

I have described an active acquisition system using a CCD. Such a system provides the means for an easy combination of data acquisition and data processing functions controlled from a high-level shell language. The active acquisition approach is valid not only for robotic telescopes, but also for normal observing facilities. It has the virtue of isolating the high-level software from the hardware-dependent layer and offers an efficient development path for new applications. Service observing, remote control and automatic maintenance may be done better through an active system.

With the present technological trends (CCD chips, computer hardware and software) soon only the CCD controller hardware and software will limit the development of active systems.

The CCD hardware should satisfy stringent speed requirements. With improving CCD onchip amplifier design, the main speed limitation comes from controllers and not from the chip (read-out noise) itself. There is an urgent need for the megapixel per-second-per-channel class, high precision controllers in the near future. These are important, not only for active systems, but also for all projects with large CCD cameras. Many controllers can handle a mosaic of 16 or even more chips, but cannot process the data at a speed which is already attainable with existing chips in many applications (high $\mathrm{S} / \mathrm{N}$ observations). Data transmission has to be as direct as possible between the controller and data-processing computer. Intermediate storage has to be avoided or it should be possible to bypass such storage.

The controller has to be integrated in an efficient development and run-time software environment. A genuine high-level programming language should be provided that enables us to describe waveforms, data flow control and electronics setup and store them in a source file. Run-time environment should provide control for rapid down-loading and should support realtime parameters which fully define the specific set-up of a current exposure/read-out mode.

\section{REFERENCES}

Blecha, A., Weber, L., Simond, G. and Queille, D. 1990 in CCDs in Astronomy, G. H. Jacoby, ed., ASP conference series, vol. 8, p. 192

Blecha, A. and Weber., L 1992 Inter-technical description, Observatoire de Genève

Brooks, P. W. 1990 Proceedings Astronomical Society of Australia, 8, no. 4, p. 377

Bruton, J. R., Hall, D. S., Boyd, L. J., Genet, R. M. and Lines, R. D. 1989 Astrophysics and Space Science, 155, 27

Burnet, M. 1976 Thesis No. 235, Ecole Polytechnique de Lausanne (French)

Filippenko, A. V., ed. 1991 Robotic Telescopes in the 1990s; ASP Conf. Series, Vol 34

Genet, R. M., Boyd, L. J., Kissell, K. E., Crawford, D. L. and Hall, D. S. 1987

PASP 99, 660 
Janesick, J., Bredhauter, R., Chandler, Ch. and Burke, B. 1988 in X-Ray Instrumentation in Astronomy. II., Proc. SPIE, 982, R. Golub, ed., p. 70

Lasker, B. M., Sturch, C. R., Mclean, B. J., Russell, J. L. and Jenkner, H. 1990 AJ 99, 2019 and 2173

Nelson, E. R. and Zeilik, M. 1990 ApJ 349, 163

Perlmutter, S., Muller, R. A., Newberg, H. J. M., Pennypacker, C. R., Sasseen, T. P. and Smith, C. K. 1991 Robotic Telescopes in the 1990's, A. V. Filppenko, ed., ASP Conf. Series, Vol 34, p. 67

Crawford, D. L. and Craine, E. R., eds. 1994 Proce. SPIE 2198, Astronomical Telescopes and Instrumentation for the 21st Century, p. 67

Queloz, D. 1995 In IAU Symposium No. 167, Advances in Array Technology and Applications, A. G. D. Philip, K. A. Janes and A. R. Upgren, eds., Kluwer Academic Press, p. 221

Rodono, M. and Cutispoto, G. 1992 A\&AS 95, 55

Richmond, M. W., Treffers, R. R. and Filippenko. A. V. 1993 PASP 105, 1164

Rufener, F. and Nicolet, B. 1988 A\&A 206, 357

Smith, R. M. 1994 Waveform Definition Language User Manual, beta release, CTIO internal document, available on request.

Tyson, N. D. and Gal, R. R. 1993 AJ 105, 1206

Vanderspek, R., Doty, J. P. and Ricker, G. R. 1991 Robotic telescopes in the 1990s, A. V. Filippenko, ed., ASP Conf. Series, Vol. 34, p. 123

Weber, L. 1993 Inter-Manuel de Reference, Observatoire de Genève (French)

Weber, L. 1992 Inter-Manuel de l'Utilisateur, Observatoire de Genève (French)

Wei, M., Chen, J. and Jiang, Z. 1990 PASP 102, 698

\section{DISCUSSION}

D'ODORICO: The approach you have presented is based on the experience you acquired with a dedicated telescope used with a CCD camera. Do you think the same type of active acquisition system could be used in the context of a large multipurpose telescope?

BLECHA: Yes indeed, the large telescope of the future will have to cope with situations where the active approach is the most appropriate. All recent experiences have shown that after the "first light" and "first astronomical result" the telescope system should continuously be upgraded and adjusted. This is exactly the field where the active approach is the most efficient one. 